\title{
OUTGASSING AND SURFACE PROPERTIES OF TIN COATED SNS RING VACUUM CHAMBERS*
}

\author{
P. He, H.C. Hseuh, M. Mapes, R. Todd ${ }^{\dagger}$, Collider-Accelerator Dept, BNL, Upton, NY 11973, USA, \\ N. Hilleret, LHC, CERN, Geneva, Switzerland
}

\begin{abstract}
The stainless steel vacuum chambers of the $248 \mathrm{~m}$ Spallation Neutron Source (SNS) accumulator ring are being coated with $\sim 100 \mathrm{~nm}$ of titanium nitride (TiN) to reduce the secondary electron yield (SEY). The coating is produced by DC magnetron sputtering using a long cathode imbedded with permanent magnets. The outgassing rates of several SNS half-cell chambers, with and without TiN coating, and before and after in-situ bake, were measured. The SEY of the coated chamber coupons were also measured with primary electron energy of 50-3000 eV. By varying the coating parameters, films of different surface roughness were produced and analyzed by scanning electron microscopy. It was found that the outgassing rate varies as a function of surface roughness of the TiN layer, and chambers with a rougher surface have a lower SEY.
\end{abstract}

\section{INTRODUCTION}

The SNS ring, with a circumference of $248 \mathrm{~m}$, consists of 4 arc sections of $34 \mathrm{~m}$ each and 4 straight sections of $28 \mathrm{~m}$ each [1]. There are 32 half cell chambers in the arc sections. Each chamber, constructed of 316L stainless steel, is approximately $4 \mathrm{~m}$ long and over $20 \mathrm{~cm}$ in diameter. Prior to TiN coating, the chambers are degassed in a vacuum furnace at $450 \mathrm{C}$ for 48 hours. A coating of $\sim 200 \mathrm{~nm}$ is subsequently laid down by DC magnetron sputtering using a long titanium cathode containing permanent magnets [2].

The operating pressure for the SNS storage ring is $<1 \times 10^{-8}$ Torr. The outgassing rate of stainless steel is well understood and confident estimates can be made for a wide variety of conditions. However, because of the required TiN coating to reduce the SEY, outgassing data for coated chambers is needed for vacuum simulations and to meet design requirements. The ring vacuum system will not be baked, so of particular interest is the outgassing data without in-situ bake.

\section{OUTGASSING MEASUREMENTS}

The outgassing rate of several SNS chambers was measured by means of the orifice method. The total surface area of each chamber is approximately 29,000 $\mathrm{cm}^{2}$. A schematic of the outgassing test setup is shown in Figure 1. The orifice size was $3.2 \mathrm{~mm}$ in diameter and $2 \mathrm{~mm}$ long. Outgassing measurements with in-situ bake were made with a $270 \mathrm{l} / \mathrm{s}$ sputter-ion pump (SIP) supplemented with a titanium sublimation pump (TSP) with a speed of $1000 \mathrm{l} / \mathrm{s}$ for $\mathrm{H}_{2}$ and base pressure of $<10^{-11}$ Torr. Inverted magnetron gauges, with a gauge correction factor of 2.4 for hydrogen [3] were placed on each side of the orifice, while a residual gas analyzer was placed on the pump side of the orifice to measure the gas composition. The entire system was baked at $250 \mathrm{C}$ for 48 hours. To measure the outgassing rate without an in-situ bake, a $150 \mathrm{l} / \mathrm{s}$ turbo-molecular pump with a base pressure of $10^{-9}$ Torr was employed in place of the SIP/TSP.

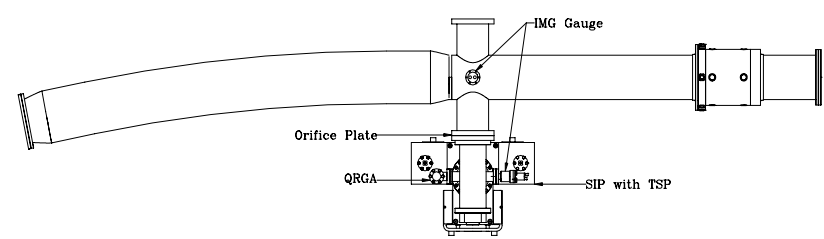

Figure 1. Schematic of outgassing measurement setup using orifice method.

A summary of the outgassing measurements is given in Table I. Outgassing rates for chambers without in-situ bake are given in $\mathrm{N}_{2}$ equivalent. Log-log plots of the outgassing rates for the chambers are given in Fig. 2.

Table I. Outgassing data of chambers under a variety of conditions.

\begin{tabular}{ccccl}
\hline $\begin{array}{c}\text { Cham } \\
\#\end{array}$ & $\begin{array}{c}\mathrm{Q}\left(\mathrm{N}_{2} \text { equiv. }\right) \\
\left(\text { Torr-1// cm }{ }^{2}\right)\end{array}$ & $\begin{array}{c}\mathrm{Q}(\mathrm{H} 2) \\
\left(\text { Torr-1/ } \mathrm{s} \mathrm{cm}^{2}\right) \\
\text { in-situ bake }\end{array}$ & $\begin{array}{c}\text { Hours under } \\
\text { vacuum } \\
\text { or post bake }\end{array}$ & Comments \\
\cline { 1 - 4 } 2A & $8.5 \mathrm{E}-12$ & $2.5 \mathrm{E}-13$ & 120 & VD, no TiN \\
9A & $3.7 \mathrm{E}-11$ & $3.0 \mathrm{E}-12$ & 120 & no VD, no TiN \\
2A & $1.2 \mathrm{E}-10$ & & 120 & VD, HP \\
3A & & $2.1 \mathrm{E}-13$ & 96 & VD, HP \\
1C & $7.1 \mathrm{E}-11$ & & 408 & VD, HP, GDT \\
7A & $2.5 \mathrm{E}-11$ & & 144 & VD, LP \\
5B & $9.6 \mathrm{E}-12$ & & 120 & VD, LP, GDT \\
5B & $2.4 \mathrm{E}-12$ & & 192 & \\
5B & & $1.9 \mathrm{E}-13$ & 72 & \\
5B & & $1.6 \mathrm{E}-13$ & 192 & \\
\hline \hline
\end{tabular}

(VD: vacuum degass, HP: coated at 4 mTorr, LP: coated at 1.5 mTorr, GDT:post glow discharge treatment)

\section{$\mathrm{H}_{2}$ Outgassing after 250C In-situ Bake}

To determine the hydrogen outgassing rate, chambers were subjected to a 48 hour $250 \mathrm{C}$ bake to remove water vapor and other adsorbed gases. An outgassing rate of $3 \times 10^{-12}$ Torr- $1 \mathrm{~s}^{-1} \mathrm{~cm}^{-2}$ was measured for chamber 9A, which was uncoated and not subjected to vacuum degassing. This outgassing rate compares favorably with

\footnotetext{
*SNS is managed by UT-Batelle, LLC, under contract DE-AC05-00OR22725 for the U.S. Department of Energy. SNS is a partnership of six national laboratories: Argonne, Brookhaven, Jefferson, Lawrence Berkely, Los Alamos, and Oak Ridge.

†rtodd@bnl.gov
} 
some previous reports of $5 \times 10^{-12}$ Torr- $1 \mathrm{~s}^{-1} \mathrm{~cm}^{-2}$ for notvacuum degassed pipes after a $300 \mathrm{C}$ bake $[4,5]$.

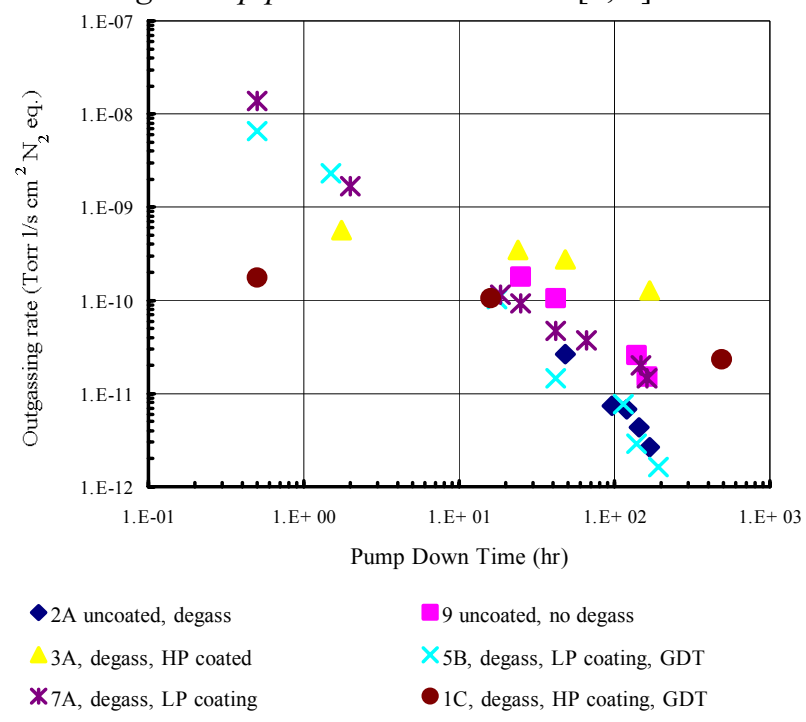

Figure 2. Outgassing rates for TiN coated and uncoated chambers without in-situ bake for a variety of coating conditions HP: 4 mTorr, LP: 1.5 mTorr, GDT: glow discharge treatment.

Subsequent to a $450 \mathrm{C}$ vacuum degass, an outgassing rate of $2.5 \times 10^{-13}$ Torr- $1 \mathrm{~s}^{-1} \mathrm{~cm}^{-2}$ was measured 120 hours after bake for un-coated chamber $2 \mathrm{~A}$, an improvement of $\sim 10 \mathrm{x}$ over chamber 9A. While diffusion theory would suggest a value as low as $4 \times 10^{-15}$ Torr- $1 \mathrm{~s}^{-1} \mathrm{~cm}^{-2}$ for our conditions [6], our measurements compare favorably with earlier measurements [4] of a lower limit of $1 \times 10^{-13}$ Torr-1 $\mathrm{s}^{-1} \mathrm{~cm}^{-2}$ for $316 \mathrm{LN}$ tubes after $950 \mathrm{C}$ vacuum fire followed by a $300 \mathrm{C}$ in-situ bake. Deviation from the theoretical values shows possible evidence of "recombination limit outgassing" explained by Moore [6].

\section{$\mathrm{H}_{2}$ Outgassing with TiN Coating}

Recent research by Saito [7] shows that TiN coating on stainless steel may act as a hydrogen permeation barrier and can reduce the hydrogen permeation rate by as much as $100 \mathrm{x}$. The outgassing rates for chambers $3 \mathrm{~A}$ and $5 \mathrm{~B}$ coated at high and low pressure, respectively, were compared to that of the uncoated chamber $2 \mathrm{~A}$. Using linear interpolation, chamber $5 \mathrm{~B}$, coated at low pressure, attained an outgassing rate of $2 \times 10^{-13}$ Torr- $1 . \mathrm{s}^{-1} \mathrm{~cm}^{-2} 120$ hours after bake, for a reduction of $\sim 30 \%$ and equilibrated at a value of $1.6 \times 10^{-13}$ Torr-1. $\mathrm{s}^{-1} \mathrm{~cm}^{-2}$. Chamber $3 \mathrm{~A}$ showed an improvement of $\sim 15 \%$, but less than that of $5 \mathrm{~B}$, likely attributed to the reduced density of the TiN film.

These results may be evidence of a permeation barrier, but not the compelling evidence we were looking for. Implicit in Saito's findings were a minimum thickness of $1 \mu \mathrm{m}$, as well stoichiometric films with few defects. Saito's substrates were electrolytically polished 304L stainless steel whereas the SNS chambers typically have a $2 \mathrm{~b}$ matt finish, at best, and a TiN nominal thickness of $200 \mathrm{~nm}$. Any one of these variables can impact the results of measurements. The $30 \%$ reduction in outgassing on chamber $5 \mathrm{~B}$ is similar to the reported value of $50 \%$ reduction by Raiteri and Calcatelli [8] on smaller samples.

\section{Outgassing without In-situ Bake}

It is evident that even without an in situ bake, there is considerable improvement in the outgassing rate of the uncoated chamber subjected to a $450 \mathrm{C}$ vacuum degass (2A over that of $9 \mathrm{~A}$ ). TiN coatings sputtered at a pressure of 4 mTorr revealed a higher than expected outgassing rate (3A). At this pressure, with a mean free path of $\sim 1$ $\mathrm{cm}$, the sputtered $\mathrm{Ti}$ was arriving at the chamber wall at a lower rate, and with less energy which produced a film with lower density and increased porosity. It was suspected that the film was very hydroscopic and RGA data showed that this gas load was almost entirely water vapor. The films produced at this pressure, were brown in color and less gold than what is typically associated with TiN. A correlation between the surface roughness of TiN films and color has been reported by Nah et-al [9]. They have determined that the more brownish TiN films are a result of increased grain size and reduced grain density.

The sputtering pressure was subsequently reduced to a level of $\sim 1.5$ mTorr, while still producing stable, uniform plasma. The resultant coating (7A) was more gold in color and found to have more acceptable outgassing rate which is a factor of $\sim 8 \mathrm{x}$ lower at 100 hours than that of the high pressure coating (3A), but still $\sim 4 \mathrm{x}$ higher than that of the uncoated chamber $(2 \mathrm{~A})$. To improve the outgassing rate further, the surface was subjected to a glow discharge treatment (GDT) before and after TiN coating. The chamber wall was bombarded with argon ions for 5 minutes for an accumulated dose of $10^{17} \mathrm{ions} / \mathrm{cm}^{2}$.
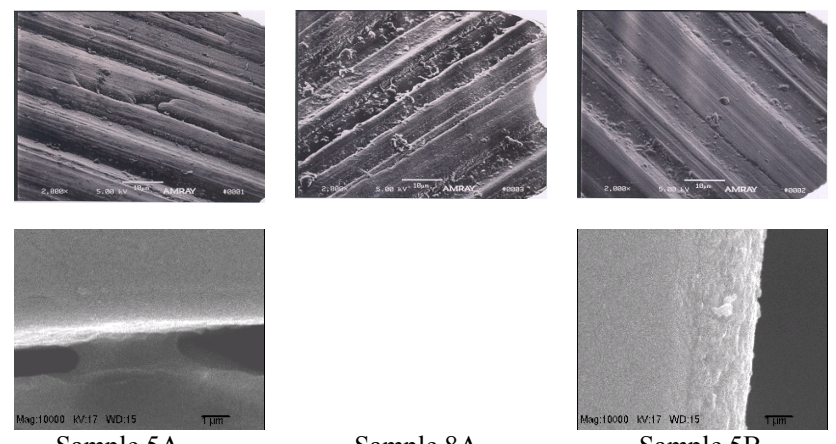

Sample 5A

Sample 8A

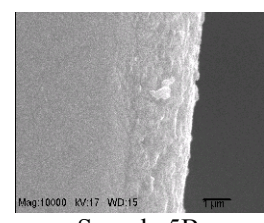

Sample 5B

Fig. 3. SEM (top) and AES (bottom) images of TiN coated surface at high pressure (5A), low pressure (8A) and low pressure with GDT (5B).

Chamber 5B was subjected to GDT and found to have an improved outgassing rate over $7 \mathrm{~A}$ by $\sim 5 \mathrm{x}$. More importantly, the outgassing rate is similar to that of an uncoated chamber $(2 \mathrm{~A})$, indicating that the quantity of water adsorbed on the TiN surface is comparable to that of uncoated stainless steel. Evidence of this can also be seen in the SEM images of the coatings (Figure 3). Sample 5A (from a chamber with identical parameters to $3 \mathrm{~A}$ ), exhibits deep voids when compared to 8A (sample from a chamber with identical parameters to $7 \mathrm{~A}$ ), while sample 5B shows further smoothing and densification. 


\section{SEY MEASUREMENTS}

SEY measurements were made on samples coated under a variety of conditions. All measurements were made as received to determine the initial SEY of the materials. Research by others have shown that significant reduction in SEY is accomplished by in-situ bakeout and ion or electron scrubbing. CERN has made extensive measurements on surfaces treated to an air bake, sputter cleaning, and oxidation treatments, and found them to be effective in lowering SEY [10]. Kirby et al has also shown that TiN has a significantly lower SEY over that of stainless steel (SS) after sputter cleaning [11].

A summary of the SEY results is given in Table II. Films produced with straight DC sputtering showed mixed SEY results and will not be discussed further. All production chambers are coated using MDC and most SEY measurements were made on coupons using this process. A summary of the SEY data is shown in Table II.

Table II. SEY data for coated and uncoated chamber coupons.

\begin{tabular}{cccl}
$\begin{array}{c}\text { Sample } \\
\#\end{array}$ & $\begin{array}{c}\text { SEY } \\
\text { Max. }\end{array}$ & $\begin{array}{c}\text { Coating } \\
\text { Parameter }\end{array}$ & Coating Parameters Legend \\
\hline \hline SS & 2.37 & 2b finish & LP- 1.5 mTorr \\
SSP & 2.49 & P & HP- 5 mTorr \\
3-Oct & 2.25 & DC/HP & GDT- Glow Discharge treatment \\
3-Jul & 2 & DC/HP & DC- Diode DC sputtering \\
19/1 & 1.89 & MDC/HP & MDC- Magnetron DC sputtering \\
20/5 & 1.99 & MDC/HP & P- Polished substrate \\
5A & 1.66 & MDC/HP & \\
2A & 2.03 & MDC/HP & \\
1C & 2.4 & MDC/HP/GDT \\
8A & 2.37 & MDC/LP & \\
4B & 2.8 & MDC/LP/GDT & \\
5B & 2.55 & MDC/LP/GDT
\end{tabular}

The effect of surface roughness on SEY has been reported [12] and demonstrated recently on copper by Baglin, et-al [13]. To measure the effect of substrate roughness, the SEY of both TiN coated and uncoated stainless steel (SS) coupons were measured. One coupon was polished, while the other retained its $2 \mathrm{~b}$ finish. As expected, the polished coupons showed a slightly higher SEY in both cases.

If the substrate roughness had a bearing on the SEY, then the deposited film's roughness will have a corresponding effect. It was found that the film with the lowest SEY was generated at a high pressure with no post GDT, while the highest SEY was on a film produced at low pressure with GDT. Unfortunately the films produced at high pressure without GDT exhibited the highest outgassing rate. In hopes of reducing the outgassing rate, while retaining the lower SEY, a film was produced at high pressure with GDT. Much to our chagrin, this film exhibited a high SEY of 2.4 and high outgassing.

In the as received state, the SEY of films generated at HP without GDT were, on average, lower than both uncoated SS and LP coated films with or without GDT. Furthermore, TiN films produced at LP with or without GDT showed no significant reduction in SEY over uncoated SS. This lack of SEY reduction in the as received state of $\mathrm{TiN}$ and $\mathrm{SS}$, has also been observed by Baglin et al [13]. It is our conclusion that the surface roughness of our TiN films is the driving force in reducing the as received $\mathrm{SEY}$, while the addition of insitu methods such as bakeout, to remove water vapor and surface gases, as well as sputter cleaning, are required to lower the achievable SEY. Measurements with in-situ bakeout and/or sputter cleaning are still required to determine our films lowest SEY.

\section{SUMMARY}

The uncoated SNS chambers exhibit lower than expected outgassing rates. The benefits of a moderate vacuum degass at $450 \mathrm{C}$ are evident. The SNS chambers coated with TiN at low pressure with glow discharge treatment have an outgassing performance comparable to uncoated chambers. There is strong evidence that TiN films produced at high pressure, regardless of glow discharge treatment, result in high water adsorption into the porous film and cause unwanted outgassing characteristics. There is some indication that the TiN film may act as a permeation barrier to hydrogen diffusing from the bulk, but more conclusive evidence is required. Finally, the surface roughness of the deposited film(s) may have bearing on the SEY of the film.

\section{ACKNOWLEDGEMENTS}

The authors would like to thank Paul Mickaliger and Joseph Sullivan of the Collider-Accelerator Vacuum Group in setting up and carrying out the experiments, and Bernard Henrist of CERN LHC Vacuum Group in performing the SEY measurements.

\section{REFERENCES}

[1] H.C. Hseuh, C.J. Liaw and M. Mapes, Proc. PAC'99, New York, USA, p1345 (1999).

[2] P. He, et-al, Proc. PAC'01, Chicago, USA, p2159 (2001).

[3] N. Peakock, HPS Corporation, (private comm..).

[4] H.C. Hseuh, X. Cui, J.Vac.Sci.Technol. A7, 2418 (1989).

[5] H.C. Hseuh et-al, Outgassing rate of RHIC warm-bore beam pipe, (unpublished) BNL, 1996

[6] B. C. Moore, J.Vac. Sci.Technol. A13, 545 (1995).

[7] K. Saito, et-al, J.Vac.Sci.Technol. A13, 556 (1995).

[8] G. Raiteri and A. Calcatelli, Vacuum, 62, 7 (2001).

[9] J.W. Nah, et-al, J.Vac.Sci.Technol. A17, 463(1999).

[10] I. Bojko, N. Hilleret, C. Scheuerlein, J. Vac. Sci Technol., A18, 972 (2000).

[11] R. E. Kirby, F. K. King, 'Secondary Electron Emission Yields From PEP-II accelerator Materials', SLAC-PUB-8212, (2000).

[12] H. Bruining, Physics and Applications of Secondary Electron Emission, Pergamon, London, 1954.

[13] V. Baglin, et-al, Proc. EPAC'00, Vienna, Austria, p217 (2000). 